\author{
Marc Murinson ${ }^{1,5 \#}$, Brandon Roth ${ }^{1,5 *}$, \\ Ehud Grosman $^{2,5}$, Dror Dicker ${ }^{3,5}$ and \\ Avishay Elis ${ }^{4,5 *}$ \\ ${ }^{1}$ New York program \\ ${ }^{2}$ Department of Medicine, Sheba Medical Center, \\ Tel-Hashomer, Israel \\ ${ }^{3}$ Department of Medicine, Hasharon Hospital, Rabin \\ Medical Center, PetachTikva, Israel \\ ${ }^{4}$ Department of Medicine, Beilinson Hospital, Rabin \\ Medical Center, Petach, Tikva, Israel \\ ${ }^{5}$ Sackler Faculty of Medicine, Tel Aviv University, \\ Tel Aviv, Israel \\ *These Authors are Equal contribution \\ Dates: Received: 11 June, 2015; Accepted: 01 \\ September, 2015; Published: 04 September, 2015 \\ *Corresponding author: Avishay Elis, MD, \\ Department of Medicine C, Beilinson Hospital, Rabin \\ Medical Center, PetachTikva 49100, Israel, Tel: \\ +972-3-9376301; Fax: +972-3-9221605; E-mail: \\ avishayel@clalit.org.il \\ www.peertechz.com
}

Keywords: Hypertension; Blood pressure

Measurement; Department of internal medicine

\section{Research Article \\ Blood Pressure Control during Hospitalization at the Department of Medicine}

\begin{abstract}
Introduction: Hypertension control has been shown to decrease morbidity and mortality. However, it is unclear whether it is worthwhile to control blood pressure (BP) in the inpatient setting. The aim of the study was to determine physicians' attitude towards the management and control of hypertension during hospitalization at internal medicine departments.

Methods: Physicians working in general medical wards were asked to answer a questionnaire regarding their views of various aspects of hypertension control and management during hospitalization in general medical wards.

Results: One hundred twenty-seven physicians responded, 58 (46\%) of them estimated that $50 \%$ of their inpatients have a diagnosis of hypertension. The majority of the respondents $(71,56 \%)$ believe that high BP should not be balanced during hospitalizations. According to the physicians report most of the BP measurements were done by nurses $(81 \%)$ or by non-certified staff $(17 \%)$. In most of the cases, the arm where BP was measured was not recorded (81\%), and measurements in both supine and standing positions were not accomplished (in $<10 \%$ of the cases according to $50 \%$ of respondents). In most of the departments $(70 \%)$ there are no specific guidelines for the treatment of hypertension. Two thirds of the physicians do not recommend antihypertensive combination medications.

Conclusions: Many patients who are hospitalized at internal medicine departments have hypertension, but there is a lack of uniformity with regard to these patients' BP measurements and treatment. The approach to BP management in internal medicine departments should be elucidated.
\end{abstract}

\section{Abbreviations}

US: United States; BP: Blood Pressure; ISIM: Israeli Society of Internal Medicine

\section{Introduction}

Hypertension is a highly common chronic medical condition worldwide. When not detected early enough or not managed adequately, it can lead to cardiovascular diseases and death. As of 2000 , nearly one billion people, approximately one-fourth of the adult population in the world had hypertension [1]. Based on data from 2007 to 2010, the American Heart Association determined that 1 in 3 U.S. Adults $\geq 20$ years of age had hypertension [2].

About half of the patients with hypertension are either not diagnosed or not well treated and not well controlled. Many patients who have persistently elevated BP in the hospital setting will continue to have high BP following hospital discharge [3]. Therefore; it may be worthwhile to identify these patients and to control their BP while they are hospitalized at internal medicine departments. However, it is difficult to manage and control hypertension in the inpatient setting. Hospitalized patients are typically hemodynamically less stable than outpatients, and tend to have a plethora of health problems not limited to hypertension alone. Furthermore, controlling hypertension often takes long periods of time, usually beyond the average hospitalization length. There are no guidelines how to manage and control BP during hospitalization at internal medicine departments
The aim of this study was to evaluate the attitude of physicians at the internal medicine departments throughout Israel towards the management and control of hypertension during hospitalization.

\section{Methods}

A questionnaire on physicians' views of various aspects of hypertension control and management during hospitalization in general medical wards was published electronically on the ISIM website between February and August 2013 (Appendix). The questionnaire evaluated views and aspects regarding the need to treat and control high BP during hospitalization, the techniques for BP measurement, and whether the department has a defined policy for evaluating and treating hypertension.

The questionnaire was completed anonymously. Data retrieved from the questionnaires were entered into an Excel (Microsoft) spreadsheet for analysis.

\section{Statistical analysis}

Data were analyzed with Statistical Package for the Social Sciences (IBM SPSS statistics) software version 15.0.

Variables were expressed as frequencies (percentage).

Results

Out of 1000 ISIM members 127 physicians responded: 40 (31\%) 
heads of departments, 40 (32\%) senior physicians, 44 (35\%) residents, and $3(2 \%)$ interns.

\section{Prevalence of hypertension in the department of medicine}

Hypertension is considered to be very common among patients in the department of medicine. 59 (46\%) physicians estimated that $50 \%$ of the inpatients have a diagnosis of hypertension, and another $35 \%$ thought that the prevalence is even higher, $>75 \%$ (Table 1A) The rate of hospitalizations for emergent hypertension was estimated by $53(42 \%)$ responders as $1-2 /$ month, another 48 (38\%) estimated it as $<1 /$ month (Table $1 \mathrm{~B}$ ). Nevertheless, a majority of the respondents (71, 56\%) thought that high BP should not be controlled during hospitalizations.

\section{Blood pressure measurement techniques}

Physicians reported that most of the BP measurements are done by nurses $(81 \%)$ or by non-certified staff $(17 \%)$. Most physicians $(81 \%)$ reported that the arm where the $\mathrm{BP}$ is measured is not recorded, and measurements in both supine and standing up positions are not done (50\% of the responders reported $<10 \%$ and $38 \%$ of the responders reported $10 \%-20 \%)$.

\section{Treatment of hypertension in the department of medicine}

In most of the departments (70\%) there are no specific guidelines for the treatment of hypertension. The attitude towards medications that were used before admission differed between routinely continuing the regimen (43\%) and renewing only the medications that were deemed necessary (52\%). Most of the physicians (74\%) expect a response to antihypertensive treatment within 2-5 days (Table 1C). Two thirds of the physicians do not recommend using antihypertensive combination medications.

Table 1: Physicians' views concerning various aspects of hypertension control and management during hospitalization in general medical wards ( $n-127)$.

A. Estimated percentage of patients with a diagnosis of hypertension that are hospitalized in internal medicine wards $n(\%)$

$25 \%$

\begin{tabular}{|l|l|}
\hline $35 \%$ & $19(15)$ \\
\hline $50 \%$ & $59(46)$ \\
\hline$>75 \%$ & $44(35)$ \\
\hline
\end{tabular}

B. Estimated number of patients that are hospitalized due to emergent hypertension (\%)

\begin{tabular}{|l|l|}
\hline$<1 /$ month & $48(38)$ \\
\hline $1-2 /$ month & $52(44)$ \\
\hline$>2$ /month & $27(21)$ \\
\hline
\end{tabular}

C. Estimated time for a response if antihypertensive treatment is changed during hospitalization $\mathbf{n}(\%)$

\begin{tabular}{|l|l|}
\hline 1 day & $19(15)$ \\
\hline $2-5$ days & $97(76)$ \\
\hline$\geq 6$ days & $11(9)$ \\
\hline
\end{tabular}

\section{Discussion}

The study results highlight the high prevalence of hypertension among hospitalized patients at the departments of medicine, the nonmethodological techniques for BP measurement as well as the various approaches towards hypertension control during hospitalization.

The survey respondents estimated that over half of the patients hospitalized on their wards have hypertension. These figures are consistent with the high percentage $(>50 \%)$ reported in the past 15 years. A review of the peer-reviewed literature on hypertension since 1976 using a predetermined search algorithm revealed that between $56.4 \%$ and $72.6 \%$ of inpatients had hypertension [3]. Two crosssectional studies in 2006 and 2009 from single-university centers in the U.S. and Italy reported hypertension prevalence of $72.6 \%$ among hospitalized patients $[4,5]$.

The majority of respondents favored the attitude that BP should not be controlled during hospitalizations. This result differs from the findings of Axon et al. in which $79.5 \%$ of resident physicians at 3 hospital sites in internal medicine, family medicine, and surgery training programs strongly believed that inpatient hypertension control was important [6]. Our opinion is that BP should be followed and treated during hospitalization, but it cannot be controlled as hospitalized patients are hemodynamically unstable and usually have other and more urgent co morbidities. In accordance with the idea that BP should be treated and controlled on an outpatient basis, the estimated rate of hospitalizations for emergent hypertension was low (1-2/month) by most of the physicians.

The majority of the respondents claimed that the arm used for measurement is not recorded, and that measurements are not done in both the supine and standing positions. The American Heart Association and the British Hypertension Society recommendations for BP measurement state that: subjects should rest for at least 5 minutes in a quiet room at ambient temperature with legs uncrossed and back and arm supported before measurements are made; smoking or drinking caffeinated beverages for 30 minutes prior to measurements should be avoided; patients should be asked not to talk during measurements; the arm should be supported on a pillow to raise it above the level of the heart; $\mathrm{BP}$ should initially be measured in both arms; two measurements should be taken on each occasion, and the initial value should be discarded if there is a $>10 \mathrm{mmHg}$ difference between the first and subsequent readings. Orthostatic changes should be assessed particularly in elderly or diabetic patients and in those with symptoms suggesting postural hypotension $[7,8]$. Based on our findings and the fact that most measurements are done by nurses or by non-certified staff at busy internal medicine wards with inadequate staffing, most of these recommendations, if not all, are not fulfilled. Since measurements are not done adequately it is not justify to decide about BP control during hospitalization.

Most respondents reported that their wards lack specific guidelines for the treatment of hypertension. Another survey in a similar population of physicians revealed that more than half of the departments have a predefined protocol for controlling diabetes mellitus during hospitalization [9]. As a result, there were variations 
regarding the attitude towards hypotensive medications that were used before admission. It differed between routinely continuing the regimen and renewing only the medications that are thought to be needed. These findings adversely affect hypertension treatment and BP control. Our policy is to continue the hypotensive regimen according to the medical situation unless there are contraindications.

The usual period to observe the maximal response after changes in antihypertensive medication is a few weeks [10-12]. Nevertheless, the overwhelming majority of respondents estimated that 2-5 days are needed to observe a response. This approach leads to poly pharmacy in BP control and to possibly high rates of adverse reaction after discharge.

Studies have shown that regimens based on antihypertensive combination medications improve patients compliance to treatment, $88 \%$ with combination versus $69 \%$ when using two medications $[13,14]$. Nevertheless, we found that two thirds of the physicians at internal medicine wards do not recommend antihypertensive combination medications.

This study had several limitations. It was a subjective survey-based study that included only 127 participants, without any interventional component. The study did, however, reveal the reality of everyday medical practice in hospitalized hypertensive patients as opposed to relying upon the ideal of care as the norm.

\section{Conclusion}

While there are a vast number of in-hospital patients with hypertension, there is a high degree of variability in the attitudes of the health care decision-makers towards hypertension during hospitalization in the department of medicine, mainly with regard to BP measurement, technique, and treatment. Further studies are required to better elucidate clear protocols for the standard of care given to in-hospital patients with hypertension.

\section{References}

1. Kearney PM, Whelton M, Reynolds K, Muntner P, Whelton PK, et al. (2005) Global burden of hypertension: analysis of worldwide data. Lancet 365: 217223.
2. Go AS, Mozaffarian D, Roger VL, Benjamin EJ, Berry JD, et al. (2013) Heart disease and stroke statistics-2013 update: a report from the American Heart Association. Circulation 127: e6-e245.

3. Axon R, Cousineau L, Egan BM (201) Prevalence and management of hypertension in the inpatient setting: a systematic review. J Hosp Med 6: 417 422.

4. Conen D, Martina B, Perruchoud AP, Leimenstoll BM (2006) High prevalence of newly detected hypertension in hospitalized patients: the value of inhospital 24-h blood pressure measurement. J Hypertens 24: 301-306.

5. Giantin V, Franchin A, Toffanello ED, Simonato M, Baccaglini K, et al. (2009) Masked and white-coat hypertension in two cohorts of elderly subjects, ambulatory and hospitalized patients. Arch Gerontol Geriatr 49 Suppl 1: 125128.

6. Axon RN, Garrell R, Pfahl K, Fisher JE, Zhao Y, et al. (2010) Attitudes and practices of resident physicians regarding hypertension in the inpatient setting. J Clin Hypertens 12: 698-705.

7. Smith $L$ (2005) New AHA recommendations for blood pressure measurement. Am Fam Physician 72:1391-1398.

8. (2005) British Hypertension Society. Fact File 12/2005. Blood pressure Measurement.

9. Elis A, Shacham-Abulafia A, Lishner M (2009) Glucose control in acutely ill patients: a survey of Israeli internal medicine departments. Isr Med Assoc J 11: $652-654$.

10. Karlberg BE, Kagedal B, Tegler L, Tolagen K (1976) Renin concentrations and effects of propranolol and spironolactone in patients with hypertension. Br Med J 1: 251-254.

11. Nishizaka MK, Zaman MA, Calhoun DA (2003) Efficacy of low-dose spironolactone in subjects with resistant hypertension. Am J Hypertens 16: 925-930.

12. Packer M, Medina N, Yushak M, Meller J (1983) Hemodynamic patterns of response during long-term captopril therapy for severe chronic heart failure. Circulation 68: 803-812.

13. Gerbino PP, Omar S (2007) Adherence patterns among patients treated with fixed dose combination versus separate antihypertensive agents. Am J Health- Syst Pharm 64: 1279-1283.

14. Dezii CM (2000) A retrospective Study of Persistence with Single Pill combination Therapy Vs. Concurrent Two pill Therapy in patients with hypertension. Manage Care 9; 2-6. 
Appendix: The questionnaire on physicians' views of various aspects of hypertension control and management during hospitalization in general medical wards. 1) In your estimation, what percentages of patients with a diagnosis of hypertension are hospitalized on your wards?

$25 \%$

$35 \%$

$50 \%$

$>75 \%$

2) In your estimation, how many inpatients on your wardare hospitalized because of emergent hypertension?

$<1 /$ month

1-2/month

$>2$ /month

3) Do you make it a point to balance hypertension during hospitalizations?

Yes

No

4) Who takes most of the blood pressure measurements on your wards?

Nurses

Doctors

Other

5) Of hospitalized patients that can stand, what percentage on your ward had blood pressure measuredboth lying down and standing up?

$<10 \%$

$10-20 \%$

$10-40 \%$

$>40 \%$

6) Is the arm where blood pressure is measured routinely recorded?

Yes

No

7) What is your policy towards hypotensivemedications that were used before admission?

Continue treatment for hypertension received before hospitalization

Renew only the drugs that appeared to be needed

Hold off on the treatment until blood pressure values are reassessed

8) If antihypertensive treatment is changedduring an admission, how long do you wait for a response?

One day

2-5 days

$>6$ days

9) Are there specific guidelines for the treatment of hypertension in your ward?

Yes

No

10) Does your department recommendthe use of antihypertensive combination medications?

Yes

No

11) What is your degree at the department of medicine?

Head of department

Senior physician

Residents

Interns or medical student

Citation: Murinson M, Roth B, Grosman E, Dicker D, Elis A (2015) Blood Pressure Control during Hospitalization at the Department of Medicine. Arch Clin 